\title{
(⿻) \\ Influence of perpendicular magnetic fields on the domain structure of permalloy microstructures grown on thin membranes
}

\author{
C. Dietrich, ${ }^{1, *}$ R. Hertel, ${ }^{2}$ M. Huber, ${ }^{1}$ D. Weiss, ${ }^{1}$ R. Schäfer, ${ }^{3}$ and J. Zweck ${ }^{1}$ \\ ${ }^{1}$ Institut für Experimentelle und Angewandte Physik, Universität Regensburg, Universitätsstraße 31, 93053 Regensburg, Germany \\ ${ }^{2}$ Institut für Festkörperforschung (IFF-9), Forschungszentrum Jülich, 52425 Jülich, Germany \\ ${ }^{3}$ Leibniz-Institut für Festkörper- und Werkstoffforschung (IFW), 01069 Dresden, Germany
}

(Received 5 March 2008; published 22 May 2008)

\begin{abstract}
Unlike the broadly studied influence of the in-plane magnetic fields on magnetic domain structures in thin-film elements, not much is known to date, on the changes of the domain structure driven by external magnetic fields applied perpendicular to the plane. Using high-resolution transmission electron microscopy studies combined with micromagnetic modeling, we investigated the effect of static, strong perpendicular fields on square and disk-shaped mesoscopic permalloy elements with a vortex flux-closure magnetization structure. In the case of square elements, we discovered an unexpected, pronounced, and reversible bending of the $90^{\circ}$ domain walls, which increases with the field strength and changes direction when the field direction is reversed. By means of micromagnetic simulations and atomic force microscopy studies, we demonstrate that this effect is connected with the minute curvatures of the sample surface. The slight curvature arises from surface tensions in the membrane on which the microstructures are fabricated.
\end{abstract}

DOI: 10.1103/PhysRevB.77.174427

PACS number(s): 75.70.Ak, 75.60.Ch, 75.75.+a

\section{INTRODUCTION}

Thin nanostructured magnetic films are promising candidates for applications in future data storage devices such as magnetic random access memory. ${ }^{1}$ Over the past years, the demand for reliable and defined bit states has given rise to extensive research on the magnetic behavior of such elements. Micron and submicron sized elements can form various magnetization patterns, ranging from uniform singledomain states to highly nonuniform patterns like vortex, ${ }^{2-7}$ flower, ${ }^{8} S$, and $C$ states. ${ }^{7,9}$ Their existence depends both on the applied magnetic field and the particular design of the element, including its shape and size. Even slight modifications in the geometry of the commonly used designs based on thin squares or disks may significantly change the magnetization patterns. Permalloy $\left(\mathrm{Ni}_{81} \mathrm{Fe}_{19}\right)$ is a frequently used material for such studies since it has only a weak uniaxial anisotropy, which can usually be neglected. The remanent state of magnetization of the thin permalloy disks shows a phase transition between a single-domain and a vortex state, depending on the geometry, namely, the diameter and the thickness. ${ }^{10}$ The vortex state is characterized by a circular flux closure with a Bloch line in its center. ${ }^{11,12}$

The ground state at remanence of sufficiently large rectangular thin-film elements is typically the Landau fluxclosure domain structure with four $90^{\circ}$ domain walls and a $180^{\circ}$ domain wall in the center. In the case of square elements, this structure degenerates to the Kittel structure, where the four $90^{\circ}$ domain walls meet in the center, forming the vortex. Four triangular domains are separated by the $90^{\circ}$ domain walls aligned along the diagonals of the square. In the center of the square, the strong but short-range exchange interaction causes an out-of-plane alignment of the magnetization that was realized in a Bloch line, known as the vortex core.

For practical applications but also for fundamental research, it is paramount to obtain a detailed understanding of the changes to the domain structure occurring in such elements when external magnetic fields are applied. Numerous studies can be found in the literature on the effect of small in-plane fields, leading to reversible and irreversible changes in the magnetic structure. The magnetization patterns of softmagnetic micron sized structures were investigated by several techniques with high lateral resolution. ${ }^{13}$ Magnetic force microscopy (MFM), ${ }^{14,15}$ spin-polarized scanning tunneling spectroscopy, ${ }^{16,17}$ and scanning electron microscopy with polarization analysis ${ }^{18,19}$ can be used for structures that were grown onto the bulk substrate, whereas Lorentz microscopy ${ }^{20,21}$ and electron holography ${ }^{22}$ require an electron-transparent substrate. In spite of these extensive studies, not much is known to date, on the influence of strong perpendicular fields on such elements. It was reported that after the application of strong external perpendicular fields, the Bloch line (vortex core) can be switched. ${ }^{23,24}$ Several studies with small external perpendicular fields were done to investigate the dynamic response of the magnetization to perpendicular field pulses. Buess and co-workers ${ }^{25-27}$ used temporally resolved magneto-optic Kerr microscopy to observe the modal structure of Py disks after the excitation by a short perpendicular field pulse. Perzlmaier et al. ${ }^{28}$ did similar studies on Py squares with the additional use of microfocus Brillouin light scattering spectroscopy. The dynamic deflection of the domain walls in Py squares due to a perpendicular field pulse was imaged with a high spatially resolved magnetic transmission $\mathrm{x}$-ray microscopy technique by Stoll et $a l .{ }^{29}$ In contrast to these very fast (in the picosecond or nanosecond range) or irreversible processes, we report here on the reversible, quasistatic deformations of magnetic structures in square and disk-shaped thin-film elements with flux-closure magnetic configuration. In the case of square elements, we observe an unexpected, well-defined bending of the $90^{\circ}$ domain walls, while in the case of disks, we observe a significantly altered equilibrium position of the vortex when small, additional in-plane fields are applied. The studies are per- 


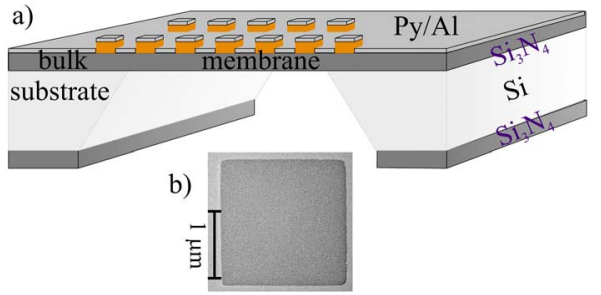

FIG. 1. (Color online) (a) Schematics of the used samples with the patterned permalloy elements evaporated on a $\mathrm{Si} / \mathrm{Si}_{3} \mathrm{~N}_{4}$ substrate. (b) Bright field TEM image of a 1.85 micron large square permalloy element, showing that the samples are polycrystalline with an edge roughness and accuracy common for this preparation technique.

formed with Lorentz microscopy and with micromagnetic simulations. The modifications of the magnetic structure occurring in strong perpendicular fields are unambiguously determined to be caused by a small curvature of the surface of the apparently flat samples.

\section{SAMPLE PREPARATION AND EXPERIMENT SETUP}

We studied the magnetization patterns of square and diskshaped permalloy $\left(\mathrm{Ni}_{81} \mathrm{Fe}_{19}\right)$ microstructures. As a substrate, we used a $30 \mathrm{~nm}$ thick electron-transparent $\mathrm{Si}_{3} \mathrm{~N}_{4}$ membrane. The $100 \times 100 \mu \mathrm{m}^{2}$ large membranes were fabricated by wet-chemical etching of a window into a $0.4 \mathrm{~mm}$ thick Si wafer ${ }^{6,30}$ (cf. Fig. 1). The patterns were defined by electron lithography using a two-layer resist with a different molecular mass. A $19 \mathrm{~nm}$ thick permalloy layer was deposited by thermal evaporation and capped by $5 \mathrm{~nm}$ aluminum to prevent oxidation. ${ }^{31}$

The magnetic domain structures were experimentally investigated using Lorentz transmission electron microscopy with a lateral magnetic resolution of about $20 \mathrm{~nm} .{ }^{13}$ The imaging was done using the Fresnel mode where the domain walls appear as dark and bright lines. ${ }^{32}$ The Philips CM30 and FEI Tecnai F30 transmission electron microscopes utilized for this study are equipped with a special imaging lens. This Lorentz lens enables us to image the sample in magnetic field free conditions. By appropriately exciting the standard objective lens, on the other hand, in situ magnetic fields can be generated. The magnetic field components, $H_{\mathrm{IP}}$ in the plane of the sample and $H_{\mathrm{OP}}$ perpendicular to the plane (outof-plane), were simply obtained by tilting the sample. ${ }^{33,34}$

\section{LORENTZ MICROSCOPY}

Lorentz microscopy was applied to image static magnetic domain structures in the square permalloy thin-film elements that were exposed to strong perpendicular fields. We observed an unexpected effect when the perpendicular field is applied, namely, the bending of the $90^{\circ}$ domain walls, which are straight lines at zero external field. The bending modifies the domain structure such as to form a "propellerlike" pattern, as shown in Fig. 2. The strength of the domain wall bending depends on the strength of the applied perpendicular field $H_{\mathrm{OP}}$ and on the sample size. Figure 2 shows the field
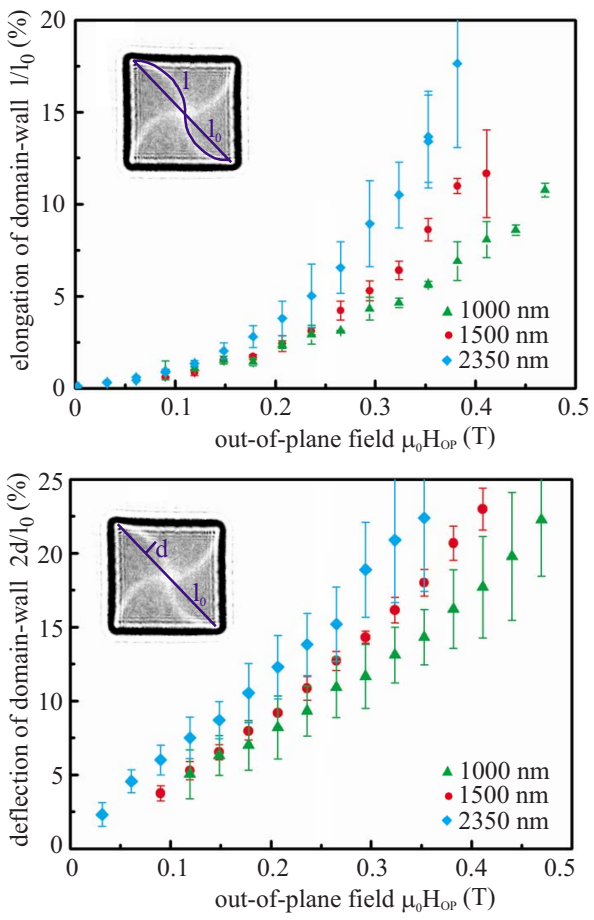

FIG. 2. (Color online) Field dependence of the bending of the domain wall for different edge lengths $(1.0,1.5$, and $2.35 \mu \mathrm{m})$. The top graph displays the relative elongation of the domain walls, whereas the bottom graph shows the relative deflection, as defined in the inset. The deflection of the domain wall increases with the increasing magnetic field and edge length. For fields up to $0.3 \mathrm{~T}$, the deflection is approximately proportional to the applied field.

dependence of the bending and the domain wall length for different edge lengths. In larger squares, the bending is more pronounced, especially for large fields, where the domain walls are $S$ shaped. The direction into which the domain walls bend depends on the sign of the applied perpendicular field.

This distortion of the domain walls is reversible, meaning that the well-known Kittel structure with straight domain walls is restored when the external field is removed.

We require a model to explain this effect. In previous studies at zero external field, similar distortions of the measured domain patterns have been assigned to image artifacts. For instance, Garcia et al. ${ }^{35}$ reported on similar perturbations in the patterned permalloy squares due to the interaction between the sample and the magnetic MFM tip, resulting in curvatures of the domain walls in the Landau flux-closure domain patterns. In our case, however, the modifications of the domain wall structure do not result from experimental artifacts. As we will show, the domain structure modifications can be explained by a deformation of the apparently flat sample, which becomes decisive only when strong perpendicular fields are applied.

\section{MODEL FOR THE DOMAIN DEFORMATION}

In an effort to understand this unexpected modification of the domain wall structures, we first performed the micromag- 
netic simulations on square elements. Interestingly, the simulations on the flat thin-film elements of corresponding size (not shown) did not yield any twist of the domain wall. On the other hand, the observations of the twisted domain structures are experimentally perfectly reproducible, showing a field-dependent twist of the domain structure in different squares of different sizes and located at various positions within the array.

To explain the effect, it is useful to point out that the observed distorted vortex structure in the presence of perpendicular fields is strongly reminiscent of the magnetization patterns on the surface of ferromagnetic cubes magnetized in a zero-field configuration known as "twisted flower state." "36 In this particular magnetic state, the vortex structure is twisted in the opposite direction on the top and bottom surfaces of a cube, cf. Fig. 12 of Ref. 36. In such cubes, the distortion of the vortex state was attributed to an additional radial component of the magnetostatic field of the sample, which gradually increases toward the border. In the cubes, this radial component has a different sign on opposite surfaces, leading to an opposite twist. Therefore, it appears that in the present case of the thin-film elements, the external, perpendicular field equally gives rise to a radial field component. (Note that without the external field, the twist is absent.)

Micromagnetic simulations indeed confirm that an additional radial field component, which radially increases in magnitude, can lead to twisted domain walls, as observed in the experiment. This is shown in Fig. 3. These micromagnetic simulations were performed using the OOMMF code. ${ }^{37}$ In agreement with the experimental sample geometry, a permalloy square of $1.85 \mu \mathrm{m}$ edge length and $19 \mathrm{~nm}$ thickness was simulated using a lateral cell size of $5 \mathrm{~nm}$. Despite the strong perpendicular fields of $303 \mathrm{mT}$ (left side of Fig. 3) and $-535 \mathrm{mT}$ (right), the sample is dominantly magnetized in plane because of the large out-of-plane demagnetizing field of more than $0.9 \mathrm{~T}^{38}$ The values of $303 \mathrm{mT}$ and $-535 \mathrm{mT}$ correspond to situations that were experimentally studied, which will be discussed later (cf. Fig. 7). By applying an external magnetic field perpendicular to the magnetic layer, the magnetization rotates nearly coherently out of the film plane as a result of minimizing the Zeeman and stray field energies. A small radial field component is applied in addition to the strong perpendicular field. This leads to a bending of the domain walls, as experimentally observed [Figs. 3(c) and 3(f)]. The radial in-plane field distribution is displayed in Figs. 3(a) and 3(b). It is assumed to be proportional to the applied field and to linearly increase with the distance from the center. In the examples shown in Fig. 2, the radial component reaches a maximum value of $13.8 \mathrm{mT}$ for the $303 \mathrm{mT}$ perpendicular field and $24.4 \mathrm{mT}$ for the perpendicular field of $-535 \mathrm{mT}$.

The simulated Fresnel images [Figs. 3(e) and 3(f)] were computed with GENIUS ${ }^{39}$ from the simulated magnetization patterns, taking into account the magnetic and electrostatic phase shifts. The electrostatic phase shift yields only to the Fresnel fringes at the sample edges under the experimentally verified (cf. next chapter) assumption of a constant thickness. For the same reason, we neglected the amplitude contrast, which is constant for the whole sample. The magnetic phase

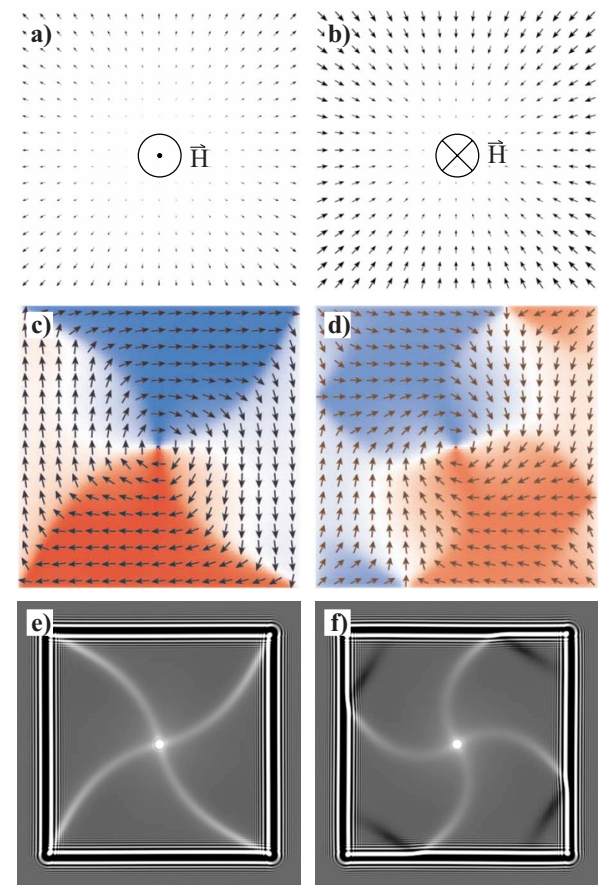

FIG. 3. (Color online) Simulations on the influence of a weak radial in-plane field on the four-domain flux-closure structure. (a) and (b) show the distribution of the radial field used in the simulations. (c) and (d) display the computed magnetization patterns, and (e) and (f) show the corresponding simulated Fresnel images. For $303 \mathrm{mT}$, the domain walls obviously bend, whereas for $-535 \mathrm{mT}$, additional magnetic domains appear at the corners. These results correspond to the experimental observations shown in Fig. 7.

shift calculated from the magnetization pattern, however, is responsible for the contrast inside the sample. The simulated images almost perfectly correspond to the experimental Fresnel images (cf. Fig. 7). The radial magnetic field shifts the position of the $90^{\circ}$ domain walls because the magnetization tends to align as far as possible with the field. From the center toward the corners, the displacement first increases (because the radial field increases) and then decreases again because the $90^{\circ}$ walls are magnetostatically pinned at the corners [Figs. 3(c) and 3(e)], leading to the observed curvature of the $90^{\circ}$ domain walls. If the radial field is strong enough, the $90^{\circ}$ domain walls can be depinned from the corners. The depinning of the domain walls from the corners results in the formation of a small domain in each corner, in which the magnetization is almost parallel to the radial field [Figs. 3(d) and 3(f)]. This is connected with the nucleation of $180^{\circ}$ head-to-head domain walls at the edges. These domain walls are comparable to the transverse domain walls in nanowires ${ }^{40}$ [cf. Fig. 3(d)], which are of great current interest in the topic of current induced domain wall motion. ${ }^{41}$

In the above simulations, a radial component was heuristically added to the external field. In the experiment, however, such a radial component cannot result directly from the applied magnetic field because, first, the external field is homogeneous to a very good approximation, particularly on the length scale of the sample size and, second, the radial component simultaneously arises for different, individual elements in the array (cf. Fig. 1). The effect must therefore be 


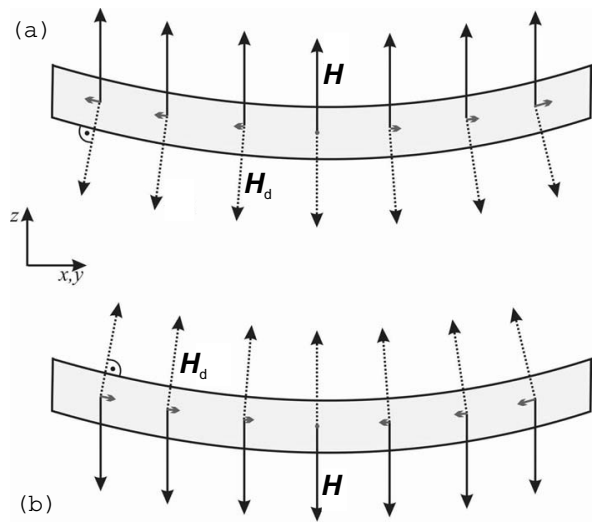

FIG. 4. (a) Schematics on the onset of a radial field component when a curved thin-film element is exposed to a strong perpendicular field. In a strong magnetic field $\boldsymbol{H}$, the magnetization partially aligns with the external field that, as a result of the curvature, is exactly perpendicular to the surface only in the center of the sample. The demagnetizing field $\boldsymbol{H}_{d}$ (dotted arrows), however, is always along the surface normal and opposite to the perpendicular magnetization component. While the demagnetizing field partially suppresses the rotation out of plane, the in-plane magnetization is directly affected by the in-plane component of $\boldsymbol{H}_{\text {net }}$. This in-plane component of $\boldsymbol{H}_{\text {net }}$ is radial (small gray arrows) and increases toward the boundary where the surface normal encloses an angle with the applied field. The sign of the radial component changes when the direction of the applied field is a reversed (b).

intrinsic to the sample. We suspected that this surprising modification of the domain structure could result from a curvature of the sample surface. The principle of this is sketched in Fig. 4. To a first approximation, the effect of the external field is to partly orient the magnetization along the field direction. In our case, there is obviously still an in-plane magnetic component that describes the domain structure but it is sufficient to consider only the magnetization component parallel to the field to explain the onset of a radial field component. In the case of a ferromagnet with a curved surface exposed to a strong perpendicular field $\boldsymbol{H}$, the local demagnetizing field $\boldsymbol{H}_{d}$ is different on the border as compared to the center. This difference does not only refer to its value ${ }^{42}$ but also to its direction. If we assume that the magnetization aligns with the field and that the sample is curved, the angle enclosed between the magnetization and the surface in the center is different from that at the border. While in the center, the magnetization is perfectly perpendicular to the plane, it is slightly tilted off the surface normal near the border. The local demagnetization field, however, is always perpendicular to the surface. It therefore only compensates for the local perpendicular component of the magnetization. This results in a net field $\boldsymbol{H}_{\text {net }}$, which increases toward the sample boundary and can be considered as the vector sum of the external and demagnetizing fields. The in-plane component $\boldsymbol{H}_{\mathrm{rad}}$ of this net field is a radial field and increases from the center to the border. The magnetization partly aligns with this radial component, leading to the observed bending of the domain wall (cf. Fig. 3). Note that this model also yields a change of the sign of the radial component when the sign of the perpendicular field is changed. Together with the experimental observation that the twist changes with the field reversal, this is consistent with the analogy of the twisted flower state.

Another, possibly simpler, description of the effect is the following: As the sample becomes curved near the boundary, the external magnetic field $\boldsymbol{H}$ can be split into an out-ofplane component $\boldsymbol{H}_{O P} \approx \boldsymbol{H}$ and a nonvanishing in-plane field component $\boldsymbol{H}_{I P}$, which obviously increases the more the surface is tilted. Due to magnetostatics, the magnetization has a tendency to remain in the sample plane so that an alignment of the magnetization with the component $\boldsymbol{H}_{\mathrm{OP}}$ of the external field significantly increases the dipolar energy. On the other hand, the magnetization is relatively free to rotate in the plane of the sample and, hence, to align with the small inplane component $\boldsymbol{H}_{\mathrm{IP}}$. Due to this different response of the magnetization to the perpendicular and the in-plane components of $\boldsymbol{H}$, the magnetic structure is distorted. This description explains that it is the imbalance in the competition between the external and dipolar fields resulting from their noncollinearity that is decisive for the twisting of the domain walls. It is not unusual that small modifications of the sample geometry, such as the described surface curvature, can strongly affect the resulting domain pattern. Experimentally, it is known that even if nominally identical specimens are investigated, the domain structure can differ either because of a multiplicity of the metastable states or due to small differences in the preparation details for different techniques. To verify our suspicion that, here, the occurrence of the inplane component and the resulting domain twist is caused by a curvature of the sample surface, we performed detailed additional experimental and simulation studies.

\section{CURVED SURFACES}

The sample thickness and the surface topography were investigated with atomic force microscopy (AFM). As shown in Fig. 1, the elements are placed on a thin membrane. Outside the window area, the membrane is supported by a $\mathrm{Si}$ bulk substrate. This allowed us to perform comparative AFM measurements on both supported and free membranes. The sample shown in Fig. 5 was identically prepared, as described before, with a nominal thickness of $15 \mathrm{~nm}$ permalloy and a capping layer of $4 \mathrm{~nm}$ aluminum. The structures deposited on the substrate supported membrane are uniformly flat with a thickness of $19 \mathrm{~nm}$ and small average roughness of about $2 \mathrm{~nm}$ (Fig. 5). By using off-axis electron holography, ${ }^{43,44}$ we could also show that the $500 \mathrm{~nm}$ large disks grown on the membrane have a uniform thickness. We conclude from these results that the thickness also remains constant for larger patterns grown on a membrane. From Fig. 5 , it is also evident that the permalloy squares fabricated onto the free standing membranes display, in fact, the suspected pronounced surface curvature. This curvature is attributed to the mechanical tension between the permalloy specimens and the $\mathrm{Si}_{3} \mathrm{~N}_{4}$ membrane due to the different thermal expansion coefficients. (The thermal expansion coefficients of the permalloy $\alpha_{\mathrm{Py}} \approx 10.5 \times 10^{-6} \mathrm{~K}^{-1}$ and $\mathrm{Si}_{3} \mathrm{~N}_{4} \quad \alpha_{\mathrm{SiN}}=2.6-3.3$ $\times 10^{-6} \mathrm{~K}^{-1}$ significantly differ ${ }^{45,46}$.) As the line profiles in Fig. 5(b) show, the specimen, together with the underlying membrane, curves downward while the freestanding mem- 


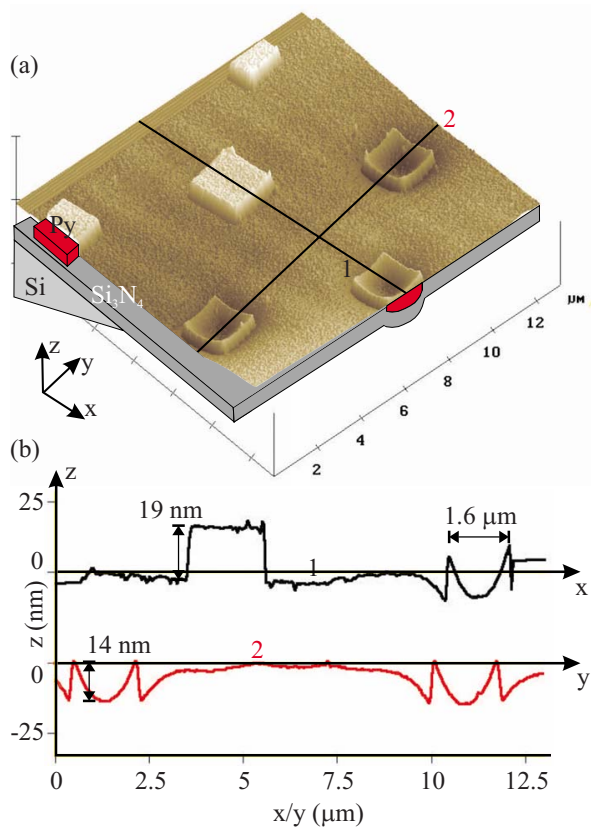

FIG. 5. (Color online) (a) AFM image showing permalloy squares deposited at the etching edge. The upper squares (bright) are still supported by the bulk substrate, whereas the lower squares (dark) are grown on the thin $\mathrm{Si}_{3} \mathrm{~N}_{4}$ membrane. (b) Line scans of the AFM image: The microstructures on the bulk substrate are uniformly flat with a constant thickness of $19 \mathrm{~nm}$. In contrast, the permalloy squares evaporated onto the membrane show a pronounced surface profile: the structures and the underlying membrane are curved. The thickness of the permalloy squares seems to be smaller for the curved film because the AFM tip fails to correctly probe the height on the downward bending membrane.

brane partially compensates for the tension between the specimens with a further deformation in the vicinity of the samples. Considering the different length scales used in the line scans displaying the variations in height as a function of the position, it becomes clear that the curvature is very small, of the order of about $10 \mathrm{~nm}$ over a distance of a micron. The depth of the curvature for elements of the same size is of the order of $2 \mathrm{~nm}$, comparable to the average roughness.

Having demonstrated that the sample surfaces are curved, and having set up a model according to which such a curvature could give rise to the radial field components in a strong perpendicular field, we shall now directly compare the micromagnetic simulations to the experimental results. It is evidently crucial to consider the small curvature of the sample surfaces in the simulation. This cannot be achieved with the OOMMF package since it relies on a finite-difference formulation of the micromagnetic equations. Due to the regular discretization grid required in the finite-difference schemes, a smooth, continuous variation of the thickness cannot be precisely modeled. We therefore used a more sophisticated code based on the finite elements. The finite-element code TetraMag is the same code that was used, for example, in Ref. 47.

The finite-element mesh used in the simulation is shown in Fig. 6. Note that in the figure the $z$ axis is scaled by a factor of ten compared to the $x$ and $y$ directions to better illustrate the parabolic profile of the surface that was as-

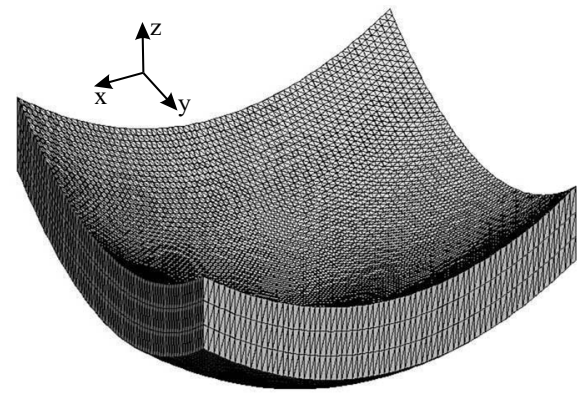

FIG. 6. Finite-element mesh used in the micromagnetic simulations to study the effect of curved surfaces. To highlight the curvature of the mesh, the dimensions in $z$ direction are scaled with a factor of ten with respect to the in-plane $x y$ axes in this image. The sample thickness is $19 \mathrm{~nm}$ while the edge length of the square is $1.85 \mu \mathrm{m}$.

sumed. A true to scale image would look nearly flat. The paraboloidal shape was used as a simple approximation of the curvature. The AFM studies reveal a variety of slightly different curvatures, which correspond well with a paraboloid. The shape of the top surface is described by

$$
z=\frac{4 s}{a^{2}} \cdot r^{2}
$$

where $r$ and $z$ are cylindrical coordinates, $s$ is the difference in height between the center and the edge of the square, and $a$ is the edge length. Hence, the difference in height between the center and the corner of the square is $2 s$. The bottom surface displays the same curvature. Hence, the thickness is homogeneous throughout the sample.

A symmetric vortex structure was chosen as the initial magnetic configuration in the simulation. A relaxation calculation (direct energy minimization using the conjugategradient method) at zero field quickly yields the well-known four-domain (Kittel-type) flux-closure domain structure [Fig. 7(a)]. A series of simulated magnetic equilibrium configurations in the curved element in the presence of perpendicular fields with various strengths is displayed in the top row of Fig. 7. Below each image, the experimental Fresnel image obtained at the corresponding field value is displayed. The perfect agreement between the simulated and experimental situations was obtained by carefully varying the value of the curvature of the finite-element mesh and comparing the simulated and the experimental results. The value of $s$ in Eq. (1), describing the curvature, is the only free parameter in the simulations. The bending of the domain walls disappears when the microstructures are assumed to be flat $(s=0)$ in the simulations. All simulation results shown in Fig. 7 have been obtained with the same finite element mesh with one value of $s$. We found the best agreement between the experiment and the simulations with $s=15 \mathrm{~nm}$ (so that the edges are $15 \mathrm{~nm}$ and the corners are about $30 \mathrm{~nm}$ above the center), which is in almost perfect agreement with the line scans obtained by the AFM. Hence, the finite-element micromagnetic simulations can be used to obtain quantitative information on the curvature of the surface. 

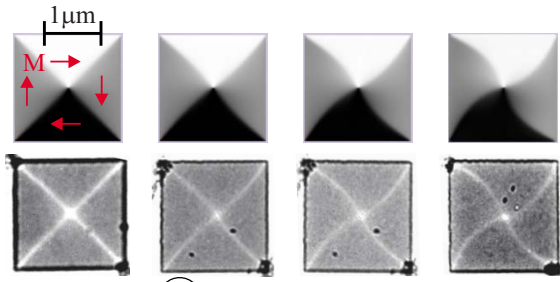

$\bigodot_{\vec{B}}$

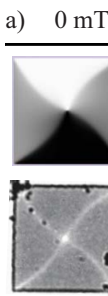

b) $88 \mathrm{mT}$

c) $188 \mathrm{mT}$

d) $303 \mathrm{mT}$
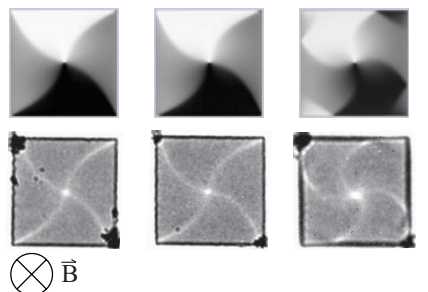

e) $-215 \mathrm{mT}$ f) $-283 \mathrm{mT}$ g) $-349 \mathrm{mT}$ h) $-535 \mathrm{mT}$

FIG. 7. (Color online) Comparison of the experiment (bottom rows) and the simulation (top rows) for permalloy squares with an edge length of $1.85 \mu \mathrm{m}$ and a thickness of $19 \mathrm{~nm}$. The images show the bending of the domain walls for different magnetic fields perpendicularly applied to the specimen. While the experimental Fresnel images (bottom) show the domain walls, the images on top show the simulated domain patterns with a grayscale coding related to the in-plane magnetization component $m_{x}$ that is indicated by the arrows in the first picture.

\section{DISKS}

The domain structure in the curved square elements under the influence of strong perpendicular fields is distorted because of the bending of the $90^{\circ}$ domain walls. The Kittel structure with four $90^{\circ}$ domain walls can be considered as a special case of a vortex structure, where the four domain walls meet in one point in the center. A typical geometry in which only the magnetic vortex states occur is disks. No domain walls are contained in these perfectly symmetric structures. Therefore, no changes in the in-plane magnetic structure under the influence of perpendicular fields are detectable with Lorentz microscopy in the Fresnel mode, even if their surface is curved. However, the simulations indicate a small and rather uniform in-plane rotation of the magnetic structure, which is not visible in the Fresnel mode where only domain walls can be imaged. Nevertheless, the curvature has also an interesting effect in these geometries, which becomes apparent under the influence of the small in-plane magnetic fields. For square elements, both effects of the domain wall bending and the modified vortex displacement superimpose. We experimentally investigated magnetic disks of thickness $t=20 \mathrm{~nm}$ and diameter $d$ ranging from 1.0 to $2.0 \mu \mathrm{m}$. The general quasistatic response of a vortex structure in a thin-film element to small external in-plane fields is the displacement of the vortex perpendicular to the field direction. By this displacement, the regions magnetized parallel to the external field increase and the regions with antiparallel magnetization shrink. Depending on the field strength, the equilibrium position of the magnetic vortex core moves

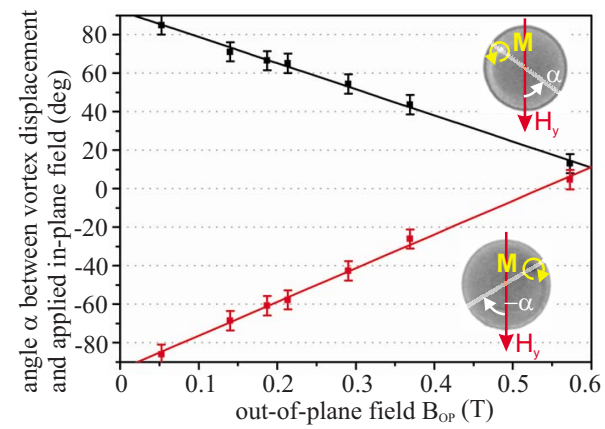

FIG. 8. (Color online) The angle between the vortex core displacement and the direction of the applied in-plane field depends on the out-of-plane field for curved disks. For small out-of-plane fields, the displacement is perpendicular to the in-plane field but it deviates from this as the perpendicular field strength is increased. The investigated permalloy disks are $19 \pm 1 \mathrm{~nm}$ thick with a diameter of $1850 \pm 20 \mathrm{~nm}$. The insets show Fresnel images taken at an applied in-plane field of $4 \mathrm{mT}$ and out-of-plane field of $0.25 \mathrm{~T}$.

from the center to the edge of the disk..$^{31,48,49}$ We studied how this behavior is altered if, additionally, a strong perpendicular magnetic field is applied. A small out-of-plane field does not affect this displacement, which is then still perpendicular to the applied in-plane field direction. Due to the small thickness compared to the lateral dimensions, the out-of-plane demagnetizing field is more than $0.9 \mathrm{~T}$ for the investigated patterns. Hence, an out-of-plane field of about 50 to $100 \mathrm{mT}$ can be considered small, whereas in-plane fields of this order already suffice to saturate the sample. The in-plane field required to shift the vortex core midway between the center and the edge is about $9 \mathrm{mT}$ for a disk with $d=1 \mu \mathrm{m}$. For stronger perpendicular fields, however, we observed that the displacement of the vortex core is no longer perpendicular to the in-plane field direction in disks grown on unsupported membranes. In these cases, the vortex is displaced by an in-plane field toward an equilibrium position along a line that encloses an angle $\alpha<90^{\circ}$ between the in-plane field. This angle depends on the strength of the out-of-plane field, as shown in Fig. 8. The sign of the angle depends on the circulation direction of the magnetization. The experimental results could be well reproduced by simulations assuming disks with a curved surface.

This further unexpected observation can equally be attributed to the radial in-plane component of the field occurring in curved elements in the case of a strong perpendicular field. As explained before, the quasistatic tendency of a vortex is to move perpendicular to the (local) field. Following the arguments described before, the vortex is shifted by the external field into a region near the boundary where it experiences a further in-plane component, i.e., the radial field resulting from the curved surface. The effective in-plane field is therefore not aligned parallel to the external field in-plane but is the vector sum of the radial and the external in-plane fields. The vortex moves to an equilibrium position that is perpendicular to the effective in-plane field, resulting in the behavior shown in Fig. 8. 


\section{CONCLUSION}

The presented experiments and simulations have revealed a high sensitivity of micromagnetic configurations on the curvature of the surface of patterned magnetic specimens. In contrast to the numerous recent publications that investigated the importance of deviations from a perfect geometry in two dimensions, namely the specimen's plane (i.e., edge roughness, ${ }^{50}$ flattened disks, ${ }^{51}$ rings, ${ }^{52}$ and disks containing one or more holes ${ }^{53,54}$ ), this study demonstrated the effects arising when the specimen is bent in the third, out-of-plane direction. Obviously, the distortions of the micromagnetic configuration are non-negligible, especially for possible applications where one has to be certain that specific micromagnetic patterns are present. These features become apparent when strong magnetic fields perpendicular to the specimen's plane are applied. One option for possible applications might be to ensure that the specimen is perfectly flat, leaving the demonstrated effects ineffective. On the other hand, the observed characteristic effects open possibilities in controlling and manipulating magnetic domain structures in a reversible, controlled fashion. This might become useful, e.g., for sensor elements, since the size of the effects is largely proportional to the strength of the external perpendicular field. *christian.dietrich@physik.uni-regensburg.de

${ }^{1}$ S. Tehrani, E. Chen, M. Durlam, M. DeHerrera, J. M. Slaughter, J. Shi, and G. Kerszykowski, J. Appl. Phys. 85, 5822 (1999).

${ }^{2}$ R. P. Cowburn, D. K. Koltsov, A. O. Adeyeye, M. E. Welland, and D. M. Tricker, Phys. Rev. Lett. 83, 1042 (1999).

${ }^{3}$ K. J. Kirk, S. McVitie, J. N. Chapman, and C. D. W. Wilkinson, J. Appl. Phys. 89, 7174 (2001).

${ }^{4}$ M. Hehn, K. Ounadjela, J.-P. Bucher, F. Rousseaux, D. Decanini, B. Bartenlian, and C. Chappert, Science 272, 1782 (1996).

${ }^{5}$ R. E. Dunin-Borkowski, M. R. McCartney, B. Kardynal, and D. J. Smith, J. Appl. Phys. 84, 374 (1998).

${ }^{6}$ J. Raabe, R. Pulwey, R. Sattler, T. Schweinböck, J. Zweck, and D. Weiss, J. Appl. Phys. 88, 4437 (2000).

${ }^{7}$ T. Shinjo, T. Okuno, R. Hassdorf, K. Shigeto, and T. Ono, Science 289, 930 (2000).

${ }^{8}$ R. Hertel and H. Kronmüller, Phys. Rev. B 60, 7366 (1999).

${ }^{9}$ M. E. Schabes and H. N. Bertram, J. Appl. Phys. 64, 1347 (1988).

${ }^{10}$ H. Hoffmann and F. Steinbauer, J. Appl. Phys. 92, 5463 (2002).

${ }^{11}$ A. Hubert, J. Phys. (France) 49, 1859 (1988).

${ }^{12}$ A. S. Arrott, B. Heinrich, and A. Aharoni, IEEE Trans. Magn. 15, 1228 (1979).

${ }^{13}$ H. Hopster and H. P. Oepen, Magnetic Microscopy of Nanostructures (Springer, Berlin, 2005).

${ }^{14}$ J. M. Garcia-Martin, A. Thiaville, J. Miltat, T. Okuno, L. Vila, and L. Piraux, J. Phys. D 37, 965 (2004).

${ }^{15}$ J. M. Garcia, A. Thiaville, and J. Miltat, J. Magn. Magn. Mater. 249, 163 (2002).

${ }^{16}$ M. Bode, M. Getzlaff, and R. Wiesendanger, Phys. Rev. Lett. 81, 4256 (1998).

${ }^{17}$ W. Wulfhekel and J. Kirschner, Appl. Phys. Lett. 75, 1944 (1999)

${ }^{18}$ K. Koike and K. Hayakawa, Jpn. J. Appl. Phys., Part 2 23, L187 (1984).

${ }^{19}$ J. Unguris, G. Hembree, R. J. Cellota, and D. T. Pierce, J. Microsc. 139, RP1 (1985).

${ }^{20}$ M. E. Hale, H. W. Fuller, and H. Rubenstein, J. Appl. Phys. 30, 789 (1959).

${ }^{21}$ H. Boersch and H. Raith, Naturwiss. 46, 574 (1959).

${ }^{22}$ A. Tonomura, T. Matsuda, H. Tanabe, N. Osakabe, J. Endo, A. Fukuhara, K. Shinagawa, and H. Fujiwara, Phys. Rev. B 25, 6799 (1982).
${ }^{23}$ R. Pulwey, M. Rahm, J. Biberger, and D. Weiss, IEEE Trans. Magn. 37, 2076 (2001).

${ }^{24}$ N. Kikuchi, S. Okamoto, O. Kitakami, Y. Shimada, S. G. Kim, Y. Otani, and K. Fukamichi, J. Appl. Phys. 90, 6548 (2001).

${ }^{25}$ M. Buess, T. Haug, M. R. Scheinfein, and C. H. Back, Phys. Rev. Lett. 94, 127205 (2005).

${ }^{26}$ M. Buess, T. P. J. Knowles, R. Höllinger, T. Haug, U. Krey, D. Weiss, D. Pescia, M. R. Scheinfein, and C. H. Back, Phys. Rev. B 71, 104415 (2005).

${ }^{27}$ M. Buess, R. Höllinger, T. Haug, K. Perzlmaier, U. Krey, D. Pescia, M. R. Scheinfein, D. Weiss, and C. H. Back, Phys. Rev. Lett. 93, 077207 (2004).

${ }^{28}$ K. Perzlmaier, M. Buess, C. H. Back, V. E. Demidov, B. Hillebrands, and S. O. Demokritov, Phys. Rev. Lett. 94, 057202 (2005).

${ }^{29}$ H. Stoll, A. Puzic, B. v. Waeyenberge, P. Fischer, J. Raabe, M. Buess, T. Haug, R. Höllinger, C. H. Back, D. Weiss, and G. Denbeaux, Appl. Phys. Lett. 84, 3328 (2004).

${ }^{30}$ J. W. M. Jacobs and J. F. C. M. Verhoeven, J. Microsc. 143, 103 (1986).

${ }^{31}$ M. Schneider, H. Hoffmann, S. Otto, T. Haug, and J. Zweck, J. Appl. Phys. 92, 1466 (2002).

${ }^{32}$ L. Reimer, Transmission Electron Microscopy (Springer, Berlin, 1997).

${ }^{33}$ J. N. Chapman, A. B. Johnston, L. J. Heyderman, S. McVitie, and W. A. P. Nicholson, IEEE Trans. Magn. 30, 4479 (1994).

${ }^{34}$ K. J. Kirk, M. R. Scheinfein, J. N. Chapman, S. McVitie, B. R. Ward, and J. G. Tennant, J. Phys. D 34, 160 (2001).

${ }^{35}$ J. M. Garcia, A. Thiaville, J. Miltat, K. J. Kirk, J. N. Chapman, and F. Alouges, Appl. Phys. Lett. 79, 656 (2001).

${ }^{36}$ R. Hertel and H. Kronmüller, J. Magn. Magn. Mater. 238, 185 (2002).

${ }^{37}$ M. J. Donahue and D. G. Porter, Object Oriented MicroMagnetic Framework (OOMMF) (http://math.nist.gov/oommf).

${ }^{38}$ M. Schneider, H. Hoffmann, and J. Zweck, Appl. Phys. Lett. 77, 2909 (2000).

${ }^{39}$ T. Haug, S. Otto, M. Schneider, and J. Zweck, Ultramicroscopy 96, 201 (2003).

${ }^{40}$ R. D. McMichael and M. J. Donahue, IEEE Trans. Magn. 33, 4167 (1997).

${ }^{41}$ G. S. D. Beach, M. Tsoi, and J. L. Erskine, J. Magn. Magn. Mater. 320, 1272 (2008). 
${ }^{42}$ J. K. Ha, R. Hertel, and J. Kirschner, Europhys. Lett. 64, 810 (2003).

${ }^{43}$ R. E. Dunin-Borkowski, M. R. McCartney, D. J. Smith, and S. S. P. Parkin, Ultramicroscopy 74, 61 (1998).

${ }^{44}$ A. Tonomura, T. Matsuda, J. Endo, T. Arii, and K. Mihama, Phys. Rev. B 34, 3397 (1986).

${ }^{45}$ Y. S. Touloukian, Thermal Expansion-Metallic Elements and Alloys (IFI/Plenum, New York, 1975).

${ }^{46}$ O. Madelung, Semiconductors: Data Handbook (Springer, Berlin, 2004).

${ }^{47}$ R. Hertel, J. Appl. Phys. 90, 5752 (2001).

${ }^{48}$ K. Yu. Guslienko, V. Novosad, Y. Otani, H. Shima, and K. Fukamichi, Appl. Phys. Lett. 78, 3848 (2001).
${ }^{49}$ T. Uhlig, M. Rahm, C. Dietrich, R. Höllinger, M. Heumann, D. Weiss, and J. Zweck, Phys. Rev. Lett. 95, 237205 (2005).

${ }^{50}$ M. Herrmann, S. McVitie, and J. N. Chapman, J. Appl. Phys. 87, 2994 (2000).

${ }^{51}$ M. Schneider, J. Liszkowski, M. Rahm, W. Wegscheider, D. Weiss, H. Hoffmann, and J. Zweck, J. Phys. D 36, 2239 (2003).

${ }^{52}$ M. Kläui, C. A. F. Vaz, J. A. C. Bland, W. Wernsdorfer, G. Faini, and E. Cambril, Appl. Phys. Lett. 81, 108 (2002).

${ }^{53}$ M. Rahm, J. Stahl, W. Wegscheider, and D. Weiss, Appl. Phys. Lett. 85, 1553 (2004).

${ }^{54}$ M. Rahm, J. Stahl, and D. Weiss, Appl. Phys. Lett. 87, 182107 (2005). 